\title{
Wheel Loader Driving Intention Recognition with Gaussian Mixture - Hidden Markov Model
}

\author{
Guoxiang $\mathrm{Cao}^{1}$, Anlin Wang ${ }^{1, \mathrm{a}}$ and Donghuan $\mathrm{Xu}^{1}$ \\ ${ }^{1}$ Research Institute of Mechanical and Electronic Engineering, School of mechanical and energy engineering, \\ Tongji University, Shanghai 200029, China
}

\begin{abstract}
Accurate recognition of driving intentions can delay upshifts under the intention of quick acceleration to maximize vehicle power performance; avoid frequent gear changes in automatic transmissions for rapid deceleration intention and make all power to flow to the bucket in the desire for fast motion of cylinders. However, due to the ambiguity of the human intentions and multiple meanings of depressing on the accelerator pedal in wheel loader, it is difficult to recognize driving intention. Nevertheless, the driver's intentions are directly reflected in the accelerator pedal, brake pedal and hydraulic valve control handle. By detecting these observable signals such as the signals of acceleration pedal's displacement and velocity, brake pedal's displacement and velocity and valve status Gaussian Mixture - Hidden Markov Model(MGHMM) can recognize the unobservable driving intentions. The experiment is done in Simulink and the results show that MGHMM can recognize driving intentions as expected.
\end{abstract}

\section{Introduction}

Wheel loader often works in construction site and the bumpy road condition brings shakes to the driver, which always fatigue the driver. In addition, frequent shifts also intensify fatigue of the driver. The application of automatic transmission in wheel loader, however, relieve driver's fatigue, but the automatic transmission makes the decisions of gearshift only when the throttle opening and vehicle speed satisfy the shift requirements. The method is effective in car, but the condition in wheel loader is different. The wheel loader has mainly two missions digging and transporting while the car only has the latter, which leads that pushing the acceleration pedal down may be not to accelerate the loader body but accelerate to tip bucket. Therefore, in order to prevent the automatic transmission from issuing wrong instructions, it is necessary to accurately recognize the driving intention. On the other hand, classic two-parameter gearshift schedule cannot avoid the problem of frequent gearshift in rapid deceleration. However, if gearshift intention can be recognized accurately, the problem of frequent gearshift in rapid deceleration would be avoid by forcing downshift.

Human behaviour intentions have uncertainties and the increasing the throttle opening has multiple meanings. The above two points make the recognition of driving intention more difficult. For the recognition of driving intention man has raised many methods. Since the problem of driving intention recognition is basically pattern recognition and condition monitoring problem, Neural Network [1-4] and other machine learning methods are suitable to solve the problems. These methods are based on a great deal of experimental data. Fuzzy Logic Control [5] is also applied in driving intention

\footnotetext{
${ }^{a}$ Corresponding author: wanganlin@tongji.edu.cn

(C) The Authors, published by EDP Sciences. This is an open access article distributed under the terms of the Creative Commons Attribution License 4.0 (http://creativecommons.org/licenses/by/4.0/).
} 
recognition. Similarly, Fuzzy Logic Control need rich experience and a large amount of time to adjust the controlled system. The above methods cannot efficiently solve the problem of wheel loader driving intention identification. However, some recent endeavours in this direction in car using a popular methodology Gaussian Mixture - Hidden Markov Models (MGHMM) [6] such as work from $\mathrm{H}$ Hou [7], H Berndt [8] et al is fruitful. It can recognize the implicit parameter of temporal data patterns and build the recognition model, and the driving intention recognition itself is a problem of Hidden Markov because human behaviour is made up of a sequence of internal "mental" states which is not directly observable, but it can be inferred from observable signals, such as acceleration pedal's displacement and velocity as well as brake pedal's displacement and velocity.

In this paper five input signals are considered including signals of acceleration pedal's displacement and velocity, brake pedal's displacement and velocity and valve status. And seven output results are Fast Slowdown, Slowdown, Natural, Keep, Acceleration, Rapid Acceleration and Valve Action. Through the trained MGHMM models driving intention can be recognized online in MATLAB Simulink.

\section{Gaussian Mixture - Hidden Markov Model}

The Hidden Markov Model is an extension and development of the Markov Model. It is a probabilistic model used to describe the statistical properties of stochastic processes. And it is a double stochastic process. The Hidden Markov Model showed as figure 1 consists of a markov chain and a general random process.

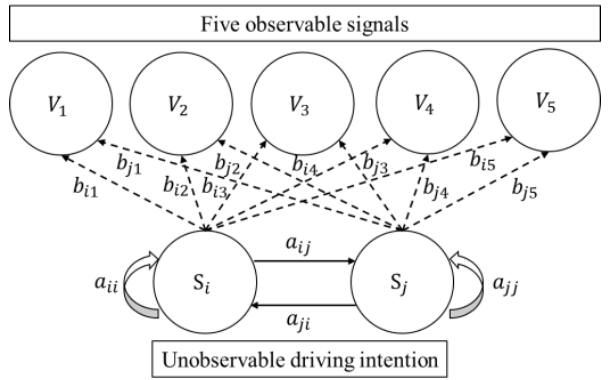

Figure 1. The Hidden Markov Model of unobservable driving intentions and observable signals

The transition probability matrix of markov chain describes the transition between two states. In general, the relationship between the state and the observed sequence is described by observation probability matrix.

HMM is defined as follows:

1. $\mathbf{X}$ represents a set of states.

$$
\mathbf{X}=\left\{S_{i} \mid 1 \leq i \leq N, i \in Z\right\}
$$

where $N$ is the number of states.

2. 0 represents a set of observable sequences.

$$
\mathbf{0}=\left\{V_{i} \mid 1 \leq i \leq M, i \in Z\right\}
$$

where $M$ is the number of observations that may be output from each state.

3. The state transition probabilities are

$$
\mathbf{A}=\left\{a_{i j}\right\}
$$

where $a_{i j}=P\left[q_{t+1}=j \mid q_{t}=i\right], 1 \leq i, j \leq N$. 
4. The observation probability matrix of State $\mathrm{j}$ is

$$
\mathbf{B}=\left\{b_{j}(k)\right\}
$$

where $b_{j}(k)=P\left[O_{t}=V_{k} \mid q_{t}=S_{j}\right], 1 \leq j \leq N, 1 \leq k \leq M . B$ is the probability of output value.

5 . The initial state distribution is

$$
\pi=\left\{\pi_{i}\right\}
$$

where $\pi_{i}=P\left[q_{i}=S_{i}\right], 1 \leq i \leq N$.

Hence HMM can be characterized by equation:

$$
\lambda=(X, O, \pi, A, B)
$$

HMM mainly solves the problem of evaluation, decoding and learning. The corresponding algorithms are Forward method, Viterbi method and Baum-Welch method.

Driving behaviour is a continuous behavior of time. For continuous observation sequences, the Gaussian Mixed Model is adopted. Then the matrix B in equation 4 is not a matrix but a probability distribution function of observation values.

$$
b_{j}(o)=\sum_{k=1}^{M} c_{j k} \mathcal{N}\left(o, \mu_{j k}, U_{j k}\right), 1 \leq j \leq N
$$

where $c$ stands for the mixture coefficient for the $k$ th mixture in the $j$ th state. $\mathcal{N}$ stands for the probability distribution function of a normal distribution with mean $\mu$ and covariance $U$ measured at observation $o$. Mixture coefficient $c$ satisfies the following constraints:

$$
\sum_{k=1}^{M} c_{j k}=1 \quad 0 \leq c_{j k} \leq 1,1 \leq j \leq N, 1 \leq k \leq M
$$

Therefore, MGHMM can be expressed as

$$
\lambda=\left(X, O, \pi, A, c, \mu, U_{j k}\right)
$$

The data to be learned is input into the model. When the model converges, it is considered that the model training has been completed.

\section{The driving intention recognition model}

\subsection{The structure of driving intention recognition model}

The driving intention of the driver in the time series is a set of decisions for each minute period under each specific work environment. That is, the driving intention recognition of each tiny period can show the change of the driver's overall driving intention and MGHMM can realize the long-term driving intention recognition by analysing short-term driving behaviour data. The figure 2 shows MGHMM recognition model structure. Considering the driver's driving intention is directly reflected in the accelerator pedal, brake pedal and hydraulic valve handle. The signals of accelerator pedal's displacement and velocity, brake pedal's displacement and velocity and hydraulic valve's switch state are chosen as input data of MGHMM. In each working cycle of the program, the seven trained models, Fast Slowdown(FS) MGHMM, Slowdown(S) MGHMM, Natural(N) MGHMM, Keep(K) MGHMM, Acceleration(A) MGHMM, Rapid Acceleration(RA) MGHMM and Valve Action(VA) MGHMM, will rapidly process the input-data with Maximum Likelihood Estimation(MLE) and the name of MGHMM corresponding the maximum value is output as the current short-term driving behaviour. 


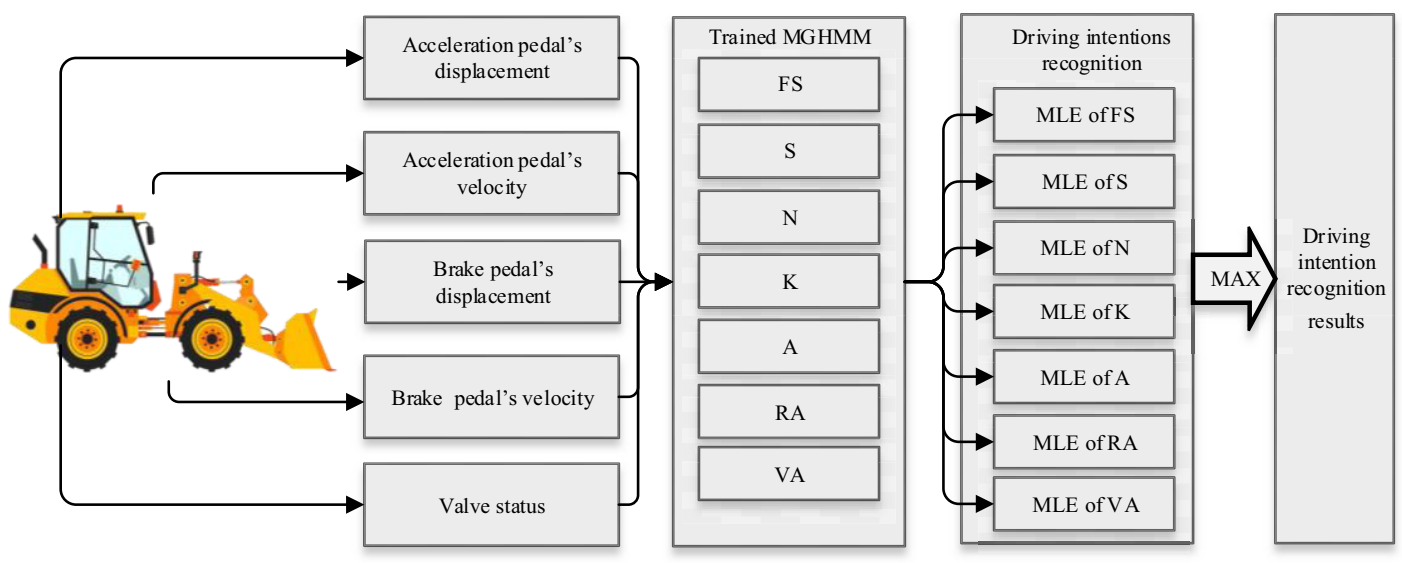

Figure 2. The structure of driving intention recognition model

\subsection{The training of MGHMM model}

All the driving behaviour MGHMMs are trained independently in MATLAB Simulink using the Baum-Welch algorithm which is an 'iterative update' algorithm to construct an HMM fitting given observation sequences. Thus, the parameters of a certain short-term driving behaviour model are optimized gradually for given learning data until convergence within a certain range. The following figure 3 shows the methods of learning data processing and MGHMMs model training processing.

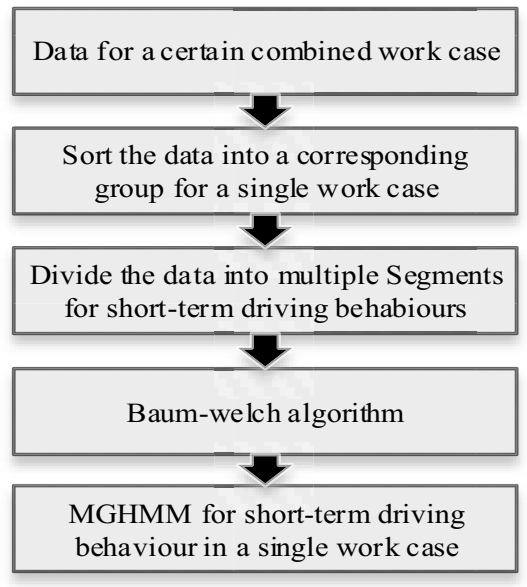

Figure 3. The method of learning data processing and MGHMMs training processing

First of all, data for a certain combined work case is collected. Then, the certain combined work case data is divided into a plurality of single work case data, and then a single work case data is divided into a plurality of time series segments. Finally, Baum-Welch algorithm is used to train each single work case MGHMM. In the experiment seven MGHMMs are finally trained, for example, When the speed of the accelerator pedal is greater than $0.2 / \mathrm{s}$, the current driving intention, in this paper, is considered to be Fast Acceleration. And all the observed signals of Fast Acceleration work cases are divided into a group for Fast Acceleration MGHMM training.

\section{Results of driving intention recognition}


After all the MGHMMs are trained, all the optimal parameters are imported into the workspace of MATLAB. The typical work conditions shown in the following figure 4 for model verification are selected. After the data to be recognized is imported into the trained models, the maximum likelihood estimators are output by the all trained models. The model name corresponding to the maximum of all MLEs is the current recognition result.

In order to improve the recognition accuracy, the input data is filtered and the recognition result is shown in the following figure 5.

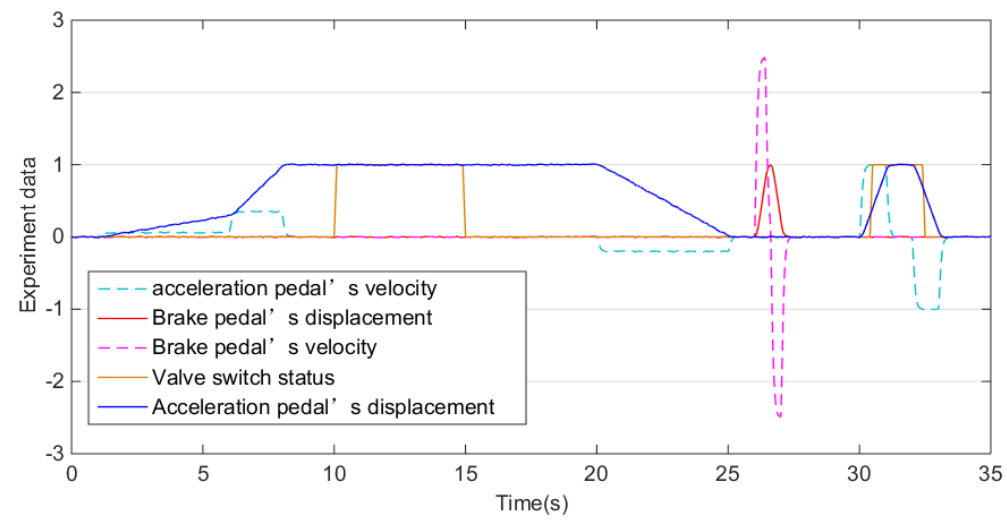

Figure 4. The typical work conditions of wheel loader

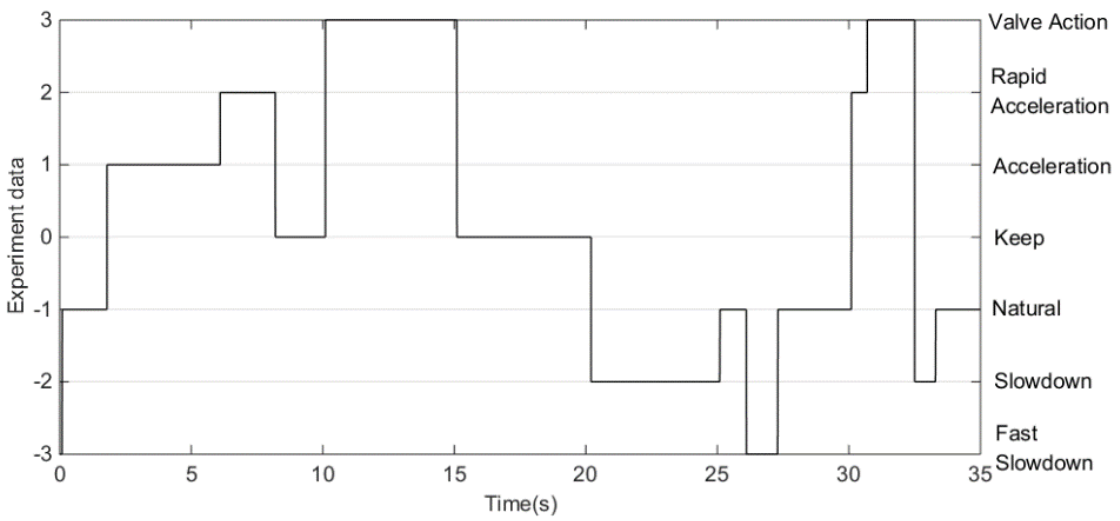

Figure 5. The results of driving intention recognition with MGHMM

The solid line in the figure 5 represents the recognition results of the driving intention. From the figure, it can be concluded that the MGHMM method can accurately identify the driver's driving intention, for example, in a period of 1 st second to 6 th second, the accelerator pedal speed is $0.06 / \mathrm{s}$ and other input signals almost zero. At the same time, the driving intention recognition result is Acceleration. In another period of 10 st second to 15 th second, the driving intention recognition result is Valve Action because the valve state is 1. Many experiments prove that the time step of $0.1 \mathrm{~s}$ can achieve the highest recognition accuracy and meet the real-time requirement of online recognition.

\section{Discussion and Conclusion}

Because of the multiple meanings of throttle, depressing the gas pedal of a car represents the desire of acceleration, while depressing the gas pedal of wheel loader may be not to accelerate vehicle but to accelerate the tipper or lifting work. Hence, it is difficult to recognize driving intention of wheel loader. However, due to the driver's intention directly reflected in the accelerator pedal, brake pedal 
and hydraulic valve control handle, MGHMM method can be used to recognize unobservable driving intentions with these observable signals. In order to improve the recognition accuracy and satisfy the real-time requirement of online recognition, the input data is divided into small fragments of $0.1 \mathrm{~s}$. Thus, this allows the TCU (Transmission Control Unit) to quickly control the transmission hydraulic system to shift gears based on the identified driving intention. Ultimately the controllability of the gearshift can be ameliorated and work efficiency improved.

In this paper, the driver's intention recognition model uses only five signals that are directly related to the driver. If further research is carried out and vehicle information and traffic information, such as current vehicle speed and current road gradient information, are considered. The MGHMM can recognize more accurately driving intentions to provide better driving assistance.

\section{Acknowledgement}

This research is supported by National Key Research and Development Program(2017YFC0704000).

\section{References}

1. Q Wang, X Tang, P Wang, et al. Driving intention identification method for hybrid vehicles based on neural network. Transactions of the Chinese Society for Agricultural Machinery, 43(8):3236(2012).

2. $\mathrm{C} \mathrm{Wu}, \mathrm{Y} \mathrm{Li}, \mathrm{X} \mathrm{Ma}$, et al. A Recognition Model for Lane Change Intention Based on Neural Network, International Conference on Transportation Information and Safety. 125-130(2013).

3. C Zong, C Wang, D Yang, et al. Driving intention identification and maneuvering behavior prediction of drivers on cornering, International Conference on Mechatronics and Automation. 4055-4060(2009).

4. R Zhang, X Yan, C Wu. A Recognition Model for Acceleration Intention of Automobile Drivers Based on Fuzzy Clustering, International Conference on Transportation Information and Safety. 1938-1947(2011).

5. J Heine, M Sylla, I Langer, et al. Algorithm for Driver Intention Detection with Fuzzy Logic and Edit Distance, IEEE, International Conference on Intelligent Transportation Systems. IEEE, 1022-1027(2015).

6. Rabiner, L.R. A tutorial on hidden Markov models and selected applications in speech recognition. Proc. IEEE,77(2):257-286(1989).

7. H Hou, L Jin, Q Niu, et al. Driver Intention Recognition Method Using Continuous Hidden Markov Model. International Journal of Computational Intelligence Systems, 4(May 2011):386393(2011).

8. H Berndt, J Emmert, K Dietmayer. Continuous Driver Intention Recognition with Hidden Markov Models, International IEEE Conference on Intelligent Transportation Systems. IEEE, 11891194(2008). 